\section{Long-term evaluation of dexamethasone intravitreal implant in vitrectomized and non-vitrectomized eyes with macular edema secondary to non-infectious uveitis}

L Pelegrín 1,2, MS de la Maza1,2, B Molins³ J Ríos ${ }^{4,5}$ and A Adán ${ }^{1,2}$

\begin{abstract}
Purpose To compare dexamethasone (DEX) intravitreal implant effect in nonvitrectomized (non-PPV) vs vitrectomized (PPV) eyes with macular edema (ME) secondary to non-infectious uveitis. Methods Medical records of patients with uveitic ME treated with DEX-intravitreal implant were reviewed. Main outcome measures were changes in central retinal thickness (CRT), best corrected visual acuity (BCVA), intraocular pressure (IOP), vitreous haze and adverse events. Statistical analysis was performed by Longitudinal Linear model using the General Estimating Equation methodology.

Results Forty-two eyes of 32 patients were included. Median follow-up time was 18 months (interquartile range (IQR): 12-24). Median CRT showed its maximum decrease at the first month in non-PPV and PPV eyes without statistically significant differences between both groups $(P=N S)$. Median Snellen BCVA, converted to logarithm (LogMAR), showed its maximum improvement at third month in both groups without statistically significant differences between them $(P=N S)$. Median IOP was higher in non-PPV eyes than in PPV eyes from third $(P=0.025)$ to 12 th month $(P=0.013)$. Vitreous haze score improved in both groups since first month and showed no differences $(P=0.706)$. Reinjection was performed in $45.2 \%$ of eyes at a median time of 5 months IQR: (5-6). Ocular hypertension $(47.6 \%)$ was the most common adverse event. Conclusions DEX-intravitreal implant for uveitic ME has similar long-term safety
\end{abstract}

profile and good response measured in terms of CRT decrease, BCVA, and vitreous haze improvement in both groups. Non-PPV eyes following DEX-intravitreal implant showed higher IOP increase than PPV eyes, showing the need for close IOP monitoring. Eye (2015) 29, 943-950; doi:10.1038/eye.2015.73; published online 22 May 2015

\section{Introduction}

Macular edema (ME) is the most frequent structural complication of intermediate, posterior uveitis, and panuveitis and is responsible for a significant amount of visual morbidity. ${ }^{1}$ Corticosteroids, administered through different ways, and anti-vascular endothelial growth factor (anti-VEGF), have long been used to treat $\mathrm{ME}$ due to non-infectious uveitis. ${ }^{2,3}$ In addition, some studies advocate performing pars plana vitrectomy in patients with uveitic $\mathrm{ME}$ as a therapeutic procedure if more conservative measures failed. ${ }^{4-6}$ A consequence of pars plana vitrectomy is that some intravitreal pharmacological treatments may have a reduced half-life in vitrectomized (PPV) eyes, possibly as a result of increased clearance. ${ }^{6}$ This decreased duration of effect may lead to a need for more frequent injections. But as approval of dexamethasone (DEX) intravitreal implant for uveitis patients, recent clinical studies have demonstrated its safety as monotherapy for the treatment of non-infectious intermediate and posterior uveitis. ${ }^{7,8}$ Its effectiveness in the treatment of uveitic ME in PPV eyes has also been reported with good results. ${ }^{9}$

\section{${ }^{1}$ Institut Clínic d'Oftalmologia, Hospital Clínic de Barcelona, Barcelona, Spain}

${ }^{2}$ Universitat de Barcelona, Barcelona, Spain

${ }^{3}$ Institut d'Investigacions Biomèdiques Agustí Pi i Sunyer (IDIBAPS), Barcelona, Spain

${ }^{4}$ Biostatistics and Data Management Core Facility, IDIBAPS, (Hospital Clinic), Barcelona, Spain

${ }^{5}$ Biostatistics Unit, Faculty of Medicine, Universitat Autònoma de Barcelona, Barcelona, Spain

Correspondence: L Pelegrín, Institut Clinic d'Oftalmologia, Hospital Casa Maternitat, Calle sabino de Arana $1,2^{\circ}$ piso, 08028 Barcelona, Spain Tel: 0034932275667 ; Fax: 0034932275662. E-mail: laurapelegrin79 @hotmail.com

Received: 2 June 2014 Accepted in revised form: 27 March 2015 Published online: 22 May 2015

This work was presented at the International Ocular Inflammation Society (IOIS), March 2014 and at the Association for Research and Vision in Ophthalmology (ARVO), May 2014. 
The purpose of this study was to compare the effectiveness of 0.7-mg DEX-intravitreal implant in noninfectious uveitic ME in eyes without previous PPV (nonPPV) vs eyes with previous PPV.

\section{Materials and methods}

We reviewed the electronic health records of all patients with uveitic ME after injection of sustained-release $0.7-\mathrm{mg}$ DEX-intravitreal implant seen at the Hospital Clinic of Barcelona from 2010 to 2013 and selected the ones with and without previous PPV. The institutional review board reviewed and approved the study protocol before initiating the study. This study was performed in accordance with the declaration of Helsinki principles. Patients included in the study were at least 18 years of age and had uveitic ME secondary to non-infectious anterior, intermediate, posterior uveitis, or panuveitis. We only have excluded patients with infectious uveitis. Ocular inflammation in these patients was managed with a stepup therapy of immunosuppressive medications. Systemic corticosteroids were administered as first step and later, immunosuppressive therapy with metotrexate, cyclosporine, azathioprine, or mycophenolate mofetile acid was administered depending on the type of ocular inflammation. In some cases, if patients still did not respond, biologic therapy was added to immunosuppressive treatment. Prior local therapies included subtenon or intravitreal triamcinolone acetonide (4 mg/0.1 ml) or intravitreal anti-VEGF bevacizumab (1.25 mg/0.05 ml).

Patients with previous glaucoma or ocular hypertension (OHT; intraocular pressure (IOP) $>23 \mathrm{~mm} \mathrm{Hg}$ ) at baseline were not excluded to analyze clinical response to DEX-intravitreal implant.

Epidemiological data including age and gender were documented. Clinical features including uveitis anatomical classification and diagnosis, laterality, previous OHT, or glaucoma, previous ME therapies, current systemic treatments, number of reinjections, and adverse events were assessed. Main outcome measures recorded were central retinal thickness (CRT), best corrected visual acuity (BCVA), IOP and vitreous haze at baseline, 2 weeks, 1, 3, 6 months and later every 6 months after first DEX-intravitreal implant injection. CRT was measured with Cirrus High Definition-Optical Coherence Tomography (Cirrus HD-OCT Model 4000, Carl Zeiss Meditec, Dublin, CA, USA; Macular Cube program), BCVA was determined with Snellen scale and was converted into logarithmic scale (LogMAR). Analysis for eyes with low visual acuity was undertaken by substituting counting fingers and hand movement with 2.0 and $2.3 \operatorname{logMAR} .{ }^{10}$ Vitreous haze was measured from 0 to $4+$ according to Standardization of Uveitis
Nomenclature (SUN). DEX-intravitreal implant was administered in a surgery room after obtaining informed consent. The eye was prepared with subconjunctival $2 \%$ lidocaine and topical 5\% povidone-iodine solution. The DEX-intravitreal implant was then injected inferotemporally according to the manufacturer's instructions. All PPV eyes had surgery previously to DEX-intravitreal implant injection.

Reinjection was performed in patients with ME relapse (CRT increase higher than 200 microns compared with lower value of CRT after first DEX-intravitreal implant and visual acuity worsening) and with previous good response to DEX-intravitreal implant.

We certify that all applicable institutional and governamental regulations concerning the ethical use of human volunteers were followed during this research.

\section{Statistical analysis}

Quantitative variables were described by median and interquartile range (IQR: percentiles 25th and 75th) and qualitative variables with absolute frequencies and percentages. Results of IOP, BCVA, or CRT are shown as estimated means and their 95\% confidence intervals (95\% CI) by group, adjusted by previous PPV, OHT, or glaucoma, reinjection of DEX-intravitreal implant during follow-up and number of treatments for OHT; in case of evaluation of CRT, time from uveitis diagnosis was also included. These estimations of effects were performed by means of Longitudinal Linear model using the General Estimating Equation (GEE) methodology to account for intra-subject correlations for visits with the assumption of first degree dependence of correlation (AR1). The GEE ${ }^{11}$ procedure extends the generalized linear model to allow for analysis of repeated measurements or other correlated observations. GEE methodology allows considering the non-independence between the two eyes of the same patient. In our study it has been considered that, as the treatment has a local effect, the result of one eye is independent from the other eye. All analyses were performed using SPSS ver.20 (IBM, Armonk, NY, USA) with a two-tailed Type I Error of 0.05 .

\section{Results}

\section{Demographics and uveitis diagnosis}

Forty-two eyes of 32 consecutive patients with noninfectious anterior, intermediate, posterior uveitis, or panuveitis with ME were included. Twenty-two women and 10 men with a median (IQR) age of 54.5 years (IQR: 3661) were analyzed. Median time since first episode of $\mathrm{ME}$ diagnosis was 6 months IQR: 4-11. Range of follow-up of patients was 6.3-35.8 months with a median of 18 months.

Most frequent anatomical locations were intermediate uveitis $(33.3 \%, 14 / 42)$, posterior uveitis $(26.2 \%, 11 / 42)$, 
panuveitis $(26.2 \%, 11 / 42)$, and anterior uveitis $(14.3 \%$, $6 / 42)$. Eight patients had idiopathic intermediate uveitis (19\% 8/42), five had birdshot chorioretinopathy $(16.7 \%$, $7 / 42)$, four patients had idiopathic panuveitis $(16.7 \%$, $7 / 42$ ), four patients had intermediate uveitis associated to multiple sclerosis $(14.3 \%, 6 / 42)$, two patients had juvenile idiopathic arthritis (JIA) associated uveitis (7.1\%, 3/42), two patients had panuveitis associated to sarcoidosis $(7.1 \%, 3 / 42)$, two patients had acute anterior uveitis associated to ankylosing spondylitis $(4.8 \%, 2 / 42)$, one patient had idiopathic retinitis, vasculitis, aneurysms, and neuroretinitis $(4.8 \%, 2 / 42)$, one patient had sympathetic ophthalmia $(2.4 \%, 1 / 42)$, one patient had posterior uveitis associated to Behçet disease $(2.4 \%, 1 / 42)$, one patient had idiopathic anterior chronic uveitis $(2.4 \%, 1 / 42)$, and one patient had idiopathic retinal vasculitis $(2.4 \%, 1 / 42)$. ME was the most common indication for treatment with DEXintravitreal implant $(61.9 \%, 26 / 42)$ as shown in Table 1.

\section{Previous local treatments, current systemic treatments, and phakic/PPV status}

At the time of the first injection of DEX-intravitreal implant, 59.4\% (19/32) of patients were on systemic treatment, which included $40.3 \%$ (13/32) on prednisone,
$28.1 \%(9 / 32)$ on immunosuppressive therapy (methotrexate (4), mycophenolate mofetil (2), cyclosporine (2), and azathioprine (1)), and 25\% (8/32) on biologic response modifier therapy (adalimumab (5), abatacept (1), golimumab (1), and beta-interferon (1)). ME was treated before DEX-intravitreal implant with intravitreal TA in $61.9 \%(26 / 42)$, periorbital TA in $45.2 \%$ $(19 / 42)$, and with intravitreal anti-VEGF in $26.2 \%(11 / 42)$. At the time of DEX-intravitreal implant injection, 78.3\% (16/42) were phakic, 13\% (21/42) were pseudophakic, and $8.7 \%(4 / 42)$ aphakic. PPV was performed in $47.6 \%$ (20/42) of eyes before DEX-intravitreal injection. ${ }^{12}$ In all cases of previous PPV, three-port PPV was performed due to the presence of epirretinal membrane.

\section{Outcome measures: CRT}

ME improvement was achieved in all eyes following DEX-intravitreal implant and this was documented by reduction of the CRT on OCT. Median CRT (95\% CI) was 591.8 microns $(234.5 ; 949)$ in non-PPV eyes and 557.3 microns $(264.6$; 850$)$ in PPV eyes at baseline; its maximum decrease was at first month, 340.6 microns $(18.5 ; 662.7)$ and 327.4 microns $(44.8 ; 610)$, respectively, (Table 2 ) and this improvement was maintained in both groups during

Table 1 Analysis of demographic and clinical data

\begin{tabular}{|c|c|c|c|c|}
\hline & \multicolumn{4}{|c|}{$P P V$} \\
\hline & Total & No & Yes & P-value \\
\hline \multicolumn{5}{|l|}{$\operatorname{Sex}$} \\
\hline Female & $31(73.8 \%)$ & $15(68.2 \%)$ & $16(80 \%)$ & $0.491^{\mathrm{a}}$ \\
\hline Male & $11(26.2 \%)$ & $7(31.8 \%)$ & $4(20 \%)$ & \\
\hline Age (years) & $55[36,61](12-83)$ & $52[38,61](12-71)$ & $55[36,62](21-83)$ & $0.935^{\mathrm{a}}$ \\
\hline Previous cataract & $4(9.5 \%)$ & $2(9.1 \%)$ & $2(10 \%)$ & $1.000^{\mathrm{a}}$ \\
\hline Previous glaucoma or $\mathrm{OHT}$ & $15(35.7 \%)$ & $3(13.6 \%)$ & $12(60 \%)$ & $0.123^{\mathrm{a}}$ \\
\hline Previous glaucoma & $8(19 \%)$ & $2(9.1 \%)$ & $6(30 \%)$ & $0.003^{\mathrm{a}}$ \\
\hline \multicolumn{5}{|l|}{ DEX-intravitreal implant indication } \\
\hline ME & $26(61.9 \%)$ & $15(68.2 \%)$ & $11(55 \%)$ & $0.336^{\mathrm{a}}$ \\
\hline Vitritis & $15(35.7 \%)$ & $6(27.3 \%)$ & $9(45 \%)$ & \\
\hline $\mathrm{ME} /$ vitritis & $1(2.4 \%)$ & $1(4.5 \%)$ & $0(0 \%)$ & \\
\hline Duration of ME (months) & $6[4,11](1-26)$ & $6[3,6](1-15)$ & $6[5,16](2-26)$ & $0.13^{\mathrm{a}}$ \\
\hline Previous use of anti-VEGF & $11(26.2 \%)$ & $5(22.7 \%)$ & $6(30 \%)$ & 0.73 \\
\hline Previous use periorbital steroids & $19(45.2 \%)$ & $10(45.5 \%)$ & $9(45 \%)$ & $1^{\mathrm{a}}$ \\
\hline Previous use intravitreal TA treatment & $26(61.9 \%)$ & $13(59.1 \%)$ & $13(65 \%)$ & $0.758^{\mathrm{a}}$ \\
\hline Adverse events during follow-up & $23(54.8 \%)$ & $10(45.5 \%)$ & $13(65 \%)$ & $0.232^{\mathrm{a}}$ \\
\hline High IOP (>21 mm Hg) & $20(47.6 \%)$ & $8(36.4 \%)$ & $12(60 \%)$ & $0.216^{\mathrm{a}}$ \\
\hline Reinjection of DEX-intravitreal implant & $19(45.2 \%)$ & $10(45.5 \%)$ & $9(45.0 \%)$ & 1.000 \\
\hline Time to second reinjection (months) & $5[5,6] ; N=19(5-7)$ & $5[5,6] ; N=10(5-7)$ & $5[5,6] ; N=9(5-7)$ & 0.905 \\
\hline
\end{tabular}

Abbreviations: DEX, dexamethasone, IOP, intraocular pressure; ME, macular edema; OHT, ocular hypertension; PPV, pars plana vitrectomy; TA, triamcinolone; VEGF, vascular endothelial growth factor.

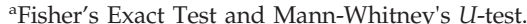

Results expressed as median [25th and 75th percentiles] and range (minimum and maximum) or absolute frequencies and percentage for quantitative or qualitative variables, respectively. 


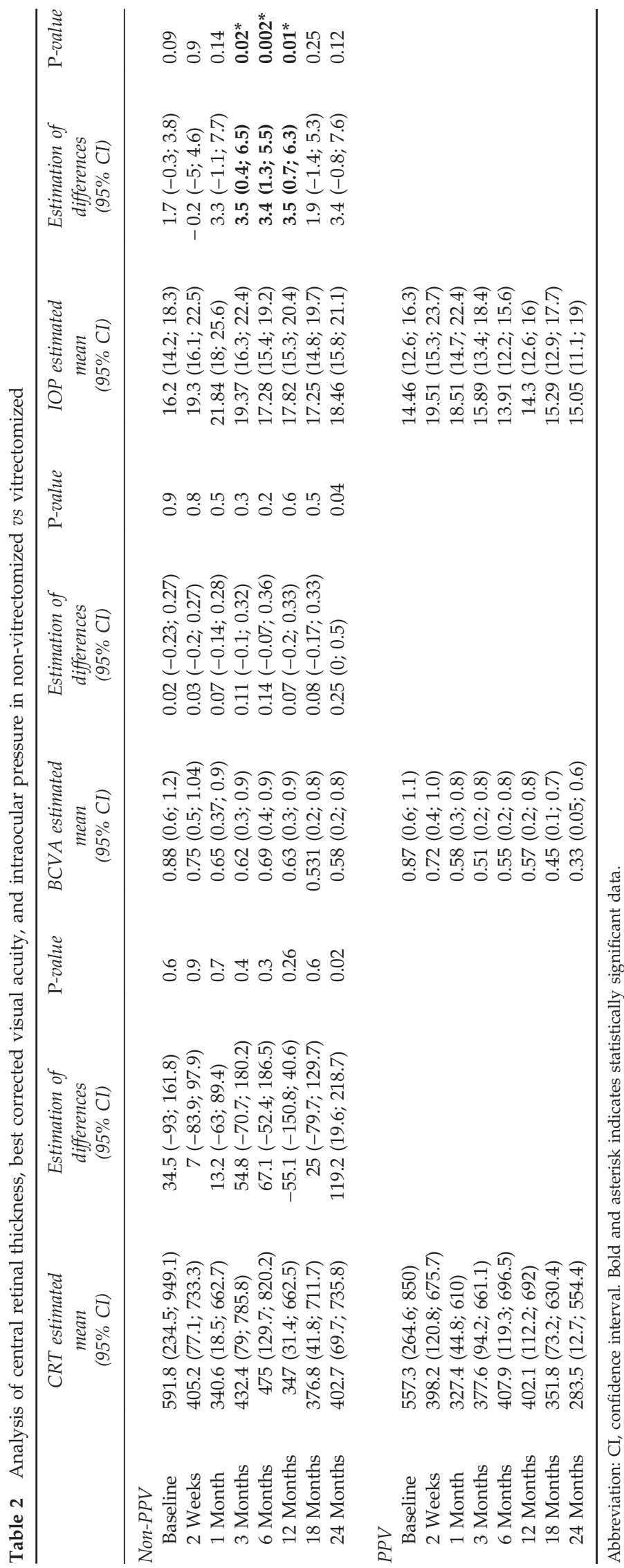


the entire follow-up. Only at 24 months of follow-up CRT analysis showed statistical differences between both groups $(P=0.019)$. CRT evolution over time after DEXintravitreal implant in non-PPV and PPV eyes is illustrated in Figure 1.

\section{Visual acuity}

BCVA improvement also was achieved in both groups. Median BCVA $(95 \%$ CI) was $0.89(0.59 ; 1.18)$ at baseline in non-PPV eyes and $0.87(0.60 ; 1.14)$ in PPV eyes; its maximum decrease was at third month; $0.62(0.33 ; 0.91)$ and $0.52(0.24 ; 0.79)$, respectively (Table 2$)$. This improvement was maintained in both groups during all follow-up and only at 24 months follow-up we observed statistical differences between both groups. BCVA evolution over time after DEX-intravitreal implant in nonPPV and PPV eyes is illustrated in Figure 2.

\section{Intraocular pressure}

Increased IOP ( $>21 \mathrm{~mm} \mathrm{Hg}$ ) was found in $47.6 \%$ of study eyes $(20 / 42)$ after DEX-intravitreal implant, including $36.4 \%(8 / 22)$ in non-PPV eyes and 60\% $(12 / 20)$ in PPV eyes $(P=0.216)$. Baseline IOP was $16.21 \mathrm{~mm} \mathrm{Hg}(14.16$; $18.26)$ in non-PPV eyes and $14.46 \mathrm{~mm} \mathrm{Hg}(12.59 ; 16.33)$ in PPV eyes. IOP analysis comparing non-PPV and PPV eyes showed statistical difference from third to 12th month. IOP was higher in non-PPV eyes showing differences of $3.5 \mathrm{~mm} \mathrm{Hg}(0.4,6.5 ; P=0.025)$ at 3 months, $3.37 \mathrm{~mm} \mathrm{Hg}$ $(1.26,5.48 ; P=0.002)$ at 6 months, and of $3.5 \mathrm{~mm} \mathrm{Hg}(0.8$, $6.3 ; P=0.013$ ) at 12 months (Table 2). IOP evolution over

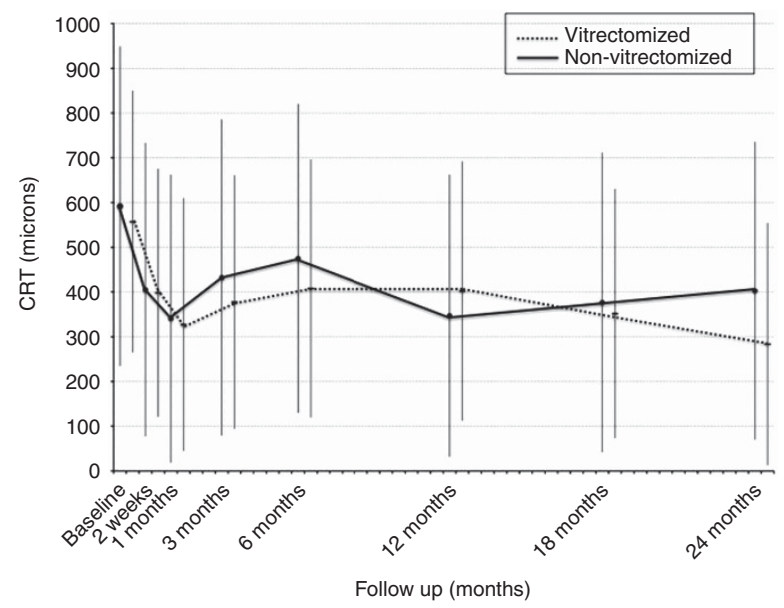

Figure 1 Central retinal thickness (CRT) evolution over time after 0.7-mg DEX-intravitreal implant in non-PPV and PPV eyes (-). CRT maximum decrease was shown at the first month and this improvement was maintained in both groups during all follow-up without statistical differences. time after DEX-intravitreal implant in non-PPV and PPV eyes is illustrated in Figure 3.

Before DEX-intravitreal implant in non-PPV eyes, glaucoma was present in two eyes (9.1\%) and OHT was present in one eye $(4.5 \%)$; all these patients needed to increase their hypotensive medication. Six eyes required hypotensive treatment de novo. Before DEX-intravitreal implant in PPV eyes, glaucoma was present in six eyes $(30 \%)$ and OHT was present in six eyes $(30 \%)$; all these patients needed to increase their hypotensive medication. Three eyes required hypotensive treatment de novo. Glaucoma previous to DEX-intravitreal implant showed statistical differences between non-PPV and PPV eyes $(P=0.003)$. From all eyes (non-PPV and PPV) only six eyes showed an IOP $>30 \mathrm{~mm} \mathrm{Hg}$ at 2 weeks after DEX-intravitreal implant injection, all of them were treated with hypotensive treatment. By week 8 , IOP was normalized in all eyes except in two eyes of one PPV patient who required filtering surgery. This patient had previous glaucoma treated with hypotensive treatment.

\section{Vitreous haze}

The vitreous haze score at baseline was $0.5+$ to $3+$ in 21 eyes (50\%). Two-step vitreous haze improvement or change from +0.5 to 0 was achieved by $66.7 \%$ of patients at 1 month, $62 \%$ of patients at 3 months, $76.2 \%$ of patients at 6 months, and $80.1 \%$ of patients at 12 months. Statistical analysis of maximum vitreous haze score changes comparing non-PPV and PPV eyes during all follow-up showed no differences $(P=0.706)$ between both groups.

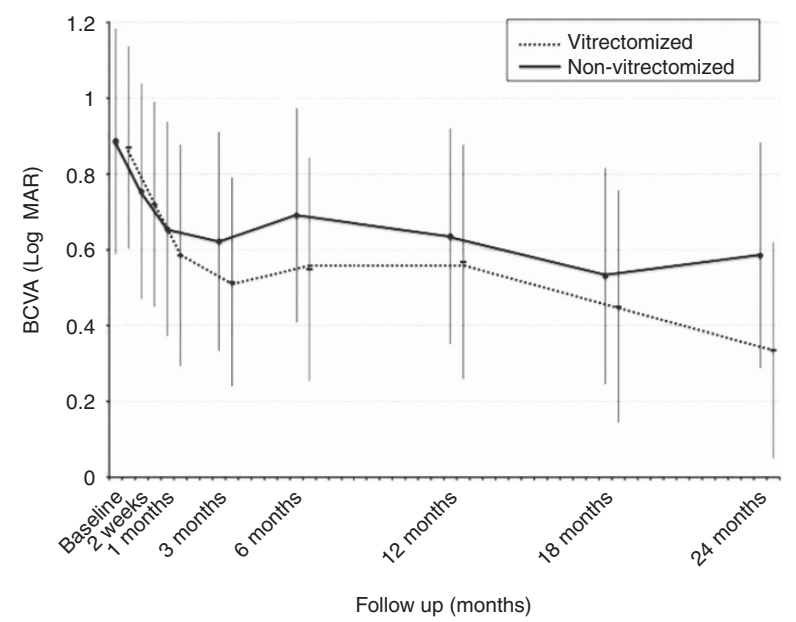

Figure 2 Best corrected visual acuity (BCVA) evolution over time after 0.7-mg DEX-intravitreal implant in non-PPV and PPV eyes (-). BCVA maximum decrease was shown at the third month and this improvement was maintained in both groups during all follow-up without statistical differences. 


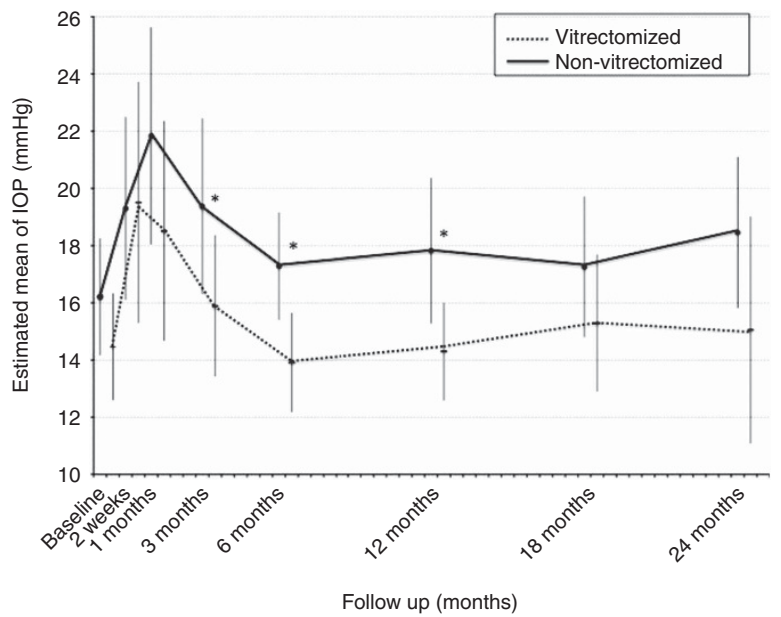

Figure 3 Intraocular pressure (IOP) evolution over time after 0.7-mg DEX-intravitreal implant in non-PPV and PPV eyes (-) IOP analysis comparing non-PPV and PPV eyes showed statistical difference from third to 12th month. Non-PPV eyes showed higher IOP compared with PPV eyes. *Statistically significant.

\section{Systemic treatment}

At the time of the first DEX-intravitreal implant, $40.3 \%$ $(13 / 32)$ of patients were on prednisone treatment and $53.1 \%(17 / 32)$ received second-line agents. According to SUN criteria for dose reduction prednisone was tapered in all cases at 1 month following the first DEX-intravitreal implant and this reduction was maintained in 78\% (7/9) of patients at 12 months. Prednisone discontinuation was achieved in $31.8 \%$ of patients at 12 months of follow-up.

\section{Adverse events}

Other ocular adverse events were transient hypotony in $7.1 \%(3 / 42)$ that resolved without any treatment in the first 4 days, vitreous hemorrhage in $7.1 \%(3 / 42)$ and anterior chamber displacement of the implant in $4.7 \%$ (2/42; one aphakic eye and one with an iris-claw intraocular lens). Moreover, four eyes showed previous cataract before DEX-intravitreal implant that progressed after DEX-intravitreal implant to the point of three of them $(7.1 \%)$ requiring cataract surgery. No DEXintravitreal implant-treated eyes developed retinal tear or detachment, or endophthalmitis during the follow-up period. At the end of follow-up, BCVA remained stable in six eyes $(14.3 \%)$ and worsened in one eye $(2.4 \%)$.

\section{Reinjections}

Twenty-three eyes (54.8\%) required only one DEXintravitreal implant to control ME. Reinjection of the implant was performed in 19 eyes (45.2\%) without differences between groups $(P=1.0)$, at a median of 5 months IQR: $5-6$,
12 eyes (28.6\%) had two injections, 5 eyes $(11.9 \%)$ had three injections and 2 eyes (4.8\%) had four injections). Further details are shown at Table 1 . No statistically significant differences were observed in the number of reinjections comparing non-PPV and PPV eyes $(P=0.812)$.

\section{Discussion}

Management of uveitic ME is a challenging issue. Antiinflammatory drugs are the mainstay of ME treatment in uveitis. Periocular or intravitreal corticosteroids, as well as intravitreal anti-VEGF, may be effective in the management of ME in uveitis. ${ }^{13-15}$ Some of our patients were previously treated with periocular and intravitreal TA or intravitreal anti-VEGF without ME improvement; DEXintravitreal implant was able to successfully improve ME in all eyes, this is probably due to the high anti-inflammatory activity of DEX that is sixfold greater than TA. ${ }^{16}$

Managing ME secondary to uveitis may be particularly challenging in eyes that have undergone prior PPV surgery because intravitreal therapies that are solutions or suspensions appear to have significantly shorter half-lives in these patients, thus requiring more frequent injections. ${ }^{17}$ DEX-intravitreal implant is a biodegradable intravitreal implant developed to provide sustained release of $700 \mu \mathrm{g}$ of preservative-free DEX that was injected in 20 PPV eyes and in 22 non-PPV eyes in our study. Our results suggest that in terms of improvement of BCVA and decrease in CRT, DEXintravitreal implant effect is similar in both groups for the treatment of uveitic ME. Following DEX-intravitreal implant, CRT decrease and vitreous haze improvement were seen at first month after and BCVA improvement was seen at third month. All those outcome measures were maintained during all follow-up in non-PPV and PPV eyes but without statistical significant differences between both groups. Similar results have been reported in DEX-intravitreal implant for treatment of $\mathrm{ME}$ secondary to central retinal vein occlusion (CRVO). ${ }^{18}$ Although the implant avoids systemic side effects, rise in IOP was the most common complication. Statistical analysis of IOP during all follow-up showed a higher IOP in non-PPV eyes compared with PPV eyes with statistically significant differences from third to 12th month. IOP increase was responsive to hypotensive treatment during 8 weeks in all eyes except in two prior glaucomatous eyes that required glaucoma surgery. Compared with previous studies, such as $\mathrm{HURON}^{7}$ study, the percentage of eyes with raised IOP in our study was higher, may be due to the differences related to the study cohorts. We did not exclude patients with previous diagnosis of glaucoma or OHT as these patients are treated with the implant in real life situations. These differences could also be explained at least in part by the 
longer follow-up period and the use of repeated DEX implants in our study. In terms of ocular inflammation (vitreous haze), we also noted improvement as early as 4 weeks and this was sustained during all follow-up.

It has been reported by Chang-Lin et al ${ }^{19}$ that vitreoretinal pharmacokinetic profiles with DEXintravitreal implant are similar between non-PPV and PPV eyes. In our study, the outcomes for both improvement in BCVA and reduction in CRT following DEX-intravitreal implant seem to be equally good in eyes that have had prior PPV compared with those having an intact vitreous. This analysis finding shows consistency with the pharmacokinetic study previously reported by Chan-Ling et al. ${ }^{19}$

Our results demonstrated the effectiveness and good safety profile of DEX-intravitreal implant in uveitic ME. Our study has some limitations and should be interpreted in the context of the retrospective design and small number of patients. As patients included in this study have been collected in a specialized uveitis unit, a selection bias should be considered.

Although similar results of comparison between non-PPV and PPV eyes have been reported in ME associated to $\mathrm{CRVO},{ }^{18}$ this series is, to the best of our knowledge, one of first to report a comparative study between non-PPV and PPV eyes with uveitic ME treated with DEX-intravitreal implant. In our series, non-PPV eyes had greater risk of developing IOP increase and therefore, should have closer IOP monitoring after DEX-intravitreal implant injection. As this is a retrospective single-center study, prospective randomized clinical trials will be needed to confirm these results.

\section{Summary}

What was known before

- The use of the dexamethasone (DEX) implant for uveitis was evaluated in the HURON trial: this study demonstrated that a single injection led to adequate control of intraocular inflammation and good visual outcomes for up to 6 months in non-infectious intermediate and posterior uveitis. Our previous report in vitrectomized (PPV) patients: DEX-intravitreal implant injection for uveitic macular edema (ME) in PPV eyes was associated with favorable visual outcomes and had an acceptable safety profile.

\section{What this study adds}

- Particularly novel: this is the first study to report a comparative study between non-PPV and PPV eyes with uveitic ME treated with DEX-intravitreal implant. Realworld experiences: it is unclear that whether the outcomes in clinical trials reflect the routine use of DEX-intravitreal implant in real situations. This study reports real-world results in an uveitis unit.

\section{Conflict of interest}

The authors declare no conflict of interest.

\section{References}

1 Lardenoye CW, van Kooij B, Rothova A. Impact of macular edema on visual acuity in uveitis. Opthalmology 2006; 113(8): 1446-1449.

2 Rothova A. Medical treatment of cystoid macular edema. Ocul Immunol Inflamm 2002; 10(4): 239-246.

3 Cervantes-Castañeda RA, Giuliari GP, Gallagher MJ, Yilmaz T, MacDonell RE, Quinones K et al. Intravitreal bevacizumab in refractory uveitic macular edema: one-year follow-up. Eur J Ophthalmol 2009; 19(4): 622-629.

4 Kiryu J, Kita M, Tanabe T, Yamashiro K, Miyamoto N, Ieky Y. Pars plana vitrectomy for cystoid macular edema secondary to sarcoid uveitis. Ophthalmology 2001; 108: 1140-1144.

5 Wiechens B, Nölle B, Reichelt JA. Pars-plana vitrectomy in cystoid macular edema associated with intermediate uveitis. Graefes Arch Clin Exp Ophthalmol 2001; 239: 474-481.

6 Heiligenhaus A, Bornfeld N, Wessing A. Long-term results of pars plana vitrectomy in the management of intermediate uveitis. Curr Opin Ophthalmol 1996; 7: 77-79.

7 Lowder C, Belfort R, Lightman S, Foster CS, Robinson MR, Schiffman RM et al. Ozurdex HURON Study Group. Dexamethasone intravitreal implant for noninfectious intermediate or posterior uveitis. Arch Ophthalmol 2011; 129(5): 545-553.

8 Tomkins-Netzer O, Taylor SR, Bar A, Lula A, Yaganti S, Talat $\mathrm{L}$ et al. Treatment with repeat dexamethasone implants results in long-term disease control in eyes with noninfectious uveitis. Ophthalmology 2014; 121(8): 1649-1654.

9 Adán A, Pelegrín L, Rey A, Llorenç V, Mesquida M, Molins B et al. Dexamethasone intravitreal implant for treatemtn of uveitic persistent cystoid macular edema in vitrectomized patients. Retina 2013; 33(7): 1435-1440.

10 Lange C, Feltgen N, Junker B, Schulze-Bonsel K, Bach M. Resolving the clinical acuity categories "hand motion" and "counting fingers" using the Freiburg Visual Acuity Test (FrACT). Graefes Arch Clin Exp Ophthalmol 2009; 247(1): 137-142.

11 Liang KY, Zeger SL. Longitudinal data analysis using generalized linear models. Biometrika 1986; 73: 13-22.

12 Jabs DA, Nussenblatt RB, Rosenbaum JT. Standardization of Uveitis Nomenclature (SUN) Working Group.

Standardization of uveitis nomenclature for reporting clinical data. Results of the first international workshop. Am J Ophthalmol 2005; 140(3): 509-516.

13 Leder HA, Jabs DA, Galor A, Dunn JP, Thorne JE. Periocular triamcinolone acetonide injections for cystoid macular edema complicating noninfectious uveitis. Am J Ophthalmol 2011; 152: 441-448.

14 Maca SM, Abela-Formanek C, Kiss CG, Sacu SG, Benesch T, Barisani-Asenbauer T. Intravitreal triamcinolone for persistent cystoid macular oedema in eyes with quiescent uveitis. Clin Experiment Ophthalmol 2009; 37(4): 389-396.

15 Young S, Larkin G, Branley M, Lightman S. Safety and efficacy of intravitreal triamcinolone for cystoid macular oedema in uveitis. Clin Exp Ophthalmol 2001; 29(1): 2-6.

16 Herrero-Vanrell R, Cardillo JA, Kuppermann BD. Clinical applications of the sustained-release dexamethasone implant for treatment of macular edema. Clin Ophthalmol 2011; 5: 139-146. 
17 Chin HS, Park TS, Moon YS, Oh JH. Difference in clearance of intravitreal triamcinolone acetonide between vitrectomized and non vitrectomized eyes. Retina 2005; 25(5): 556-560.

18 Shaikh AH, Petersen MR, Sisk RA, Foster RE, Riemann CD, Miller DM. Comparative effectiveness of the dexamethasone intravitreal implant in vitrectomized and non-vitrectomized eyes with macular edema secondary to central retinal vein occlusion. Ophthalmic Surg Lasers Imaging Retina 2013; 44(1): 28-33.

19 Chang-Lin JE, Burke JA, Peng Q, Lin T, Orilla WC, Ghosn CR et al. Pharmacokinetics of a sustained-release dexamethasone intravitreal implant in vitrectomized and nonvitrectomized eyes. Invest Ophthalmol Vis Sci 2011; 52(7): 4605-4609. 